\title{
What's the appropriate adjustment when an approved drug is in short supply?
}

\begin{abstract}
T he Rosenfeld laboratory was researching the efficacy in rodents of certain new synthetic opioids when a nationwide shortage of buprenorphine hydrochloride occurred. Buprenorphine was being used as a negative experimental control because previous research demonstrated that the drug was not effective in alleviating the induced neuropathic pain that was being studied. "Not a problem," said Lonny Lyons, one of the post-docs in the lab, "we have USP buprenorphine reference standard from some earlier work and we'll just use it instead of the commercial drug." But when the request for the change reached the attending veterinarian for concurrence via the Veterinary Verification and Consultation (VVC) method, the veterinarian said she was not able to concur with the request because
\end{abstract}

the literature provided by the vendor of the reference standard said that it was not intended to be used as a drug.

Still undaunted, the lab requested that it be allowed to use its recently outdated commercial buprenorphine hydrochloride, and they provided the IACUC with literature citations that claimed most drugs can be used well past their expiration dates without a loss of efficacy. Accompanying the request was a note from Dr. Rosenfeld explaining that she could not substitute a different negative control drug in the middle of her research. The veterinarian referred the request to the full committee which was meeting in a few days. At that meeting she said that upon reflection, she did not think that either the outdated buprenorphine or the buprenorphine reference standard would create an animal welfare issue, but she was reluctant to verify the use of either of those products without IACUC input. She added that she had tried borrowing in-date buprenorphine from nearby colleagues, but her attempts were unsuccessful.

Do you think that the IACUC should agree with the use of the outdated drug, the reference standard, or take an entirely different approach? Was the VVC method being used appropriately?

\section{Jerald Silverman}

University of Massachusetts Medical School,

Worcester, Massachusetts, USA.

e-mail: Jerald.Silverman@umassmed.edu

Published online: 23 May 2018

https://doi.org/10.1038/s41684-018-0069-9

\section{Achieving the desired outcome}

T he scenario states that buprenorphine is administered to rodents as a negative control in a study evaluating the efficacy of certain new synthetic opioids; however, a nationwide shortage of buprenorphine has occurred. The IACUC is faced with the challenge of trying to decide if it is acceptable for Dr. Rosenfeld to use outdated

buprenorphine or a buprenorphine reference standard to complete her study. The IACUC's policy on veterinary verification and consultation (VVC) is not provided in this scenario, but it seems unlikely that the policy would address the use of either a reference standard or an expired drug, so the Attending Veterinarian was correct in referring the request to the full committee.

An understanding of what a drug reference standard is would be helpful to the IACUC in evaluating this problem. "A reference standard is a substance prepared for use as the standard in an assay, identification, or purity test and should have a quality appropriate for its use ${ }^{1}$." As noted in the scenario, reference standards are not intended to be used as a drug. The United States Pharmacopeia (USP) states, "USP Reference Standards are not for use in humans or animals as drugs or medical devices. They are intended for test and assay use only as per their associated USP compendial application(s) ${ }^{2}$." Given this information, the use of the buprenorphine reference standard in Dr. Rosenfeld's study would not be an acceptable solution. Furthermore, the drug reference standard may yield results that differ from those obtained using the commercial buprenorphine product.

Today's standard of veterinary care does not recognize the use of expired drugs, biologics, and medical supplies as an acceptable practice ${ }^{3}$. However, performance standards may be used to address a variety of issues related to humane animal care and use. The Guide states, "Performance standard means a standard or guideline that, while describing a desired outcome, provides flexibility in achieving this outcome by granting discretion to those responsible for managing the animal care and use program, the researcher, and the IACUC. The performance approach requires professional input, sound judgment, and a team approach to achieve specific goals... Performance standards can be advantageous because they accommodate the consideration of many variables... so that implementation can be best tailored to meet the recommendations in the Guide ${ }^{4}$." OLAW's position on performance standards says that"a welldeveloped performance standard meets the following criteria: supports scientific objectives, supports the health and welfare of the animal; includes a justified performance index; and has associated outcome criteria ${ }^{5 . "}$ In this case, the IACUC and Dr. Rosenfeld could develop a performance standard for the use of an expired drug stating that it is essential to the experiment; it is not (currently) commercially available; it has been verified to be safe and effective for use in animals through laboratory verification of potency, sterility, stability, and other parameters; and the results obtained using the expired drug are expected to be consistent with previously collected data that used the commercial buprenorphine product. The IACUC may also want to request documentation be obtained from the manufacturer of the expired buprenorphine that the product can be used past its expiration date for a specified length of time without a loss of efficacy.

If all the performance standard criteria are met, the IACUC could vote to approve an amendment on the use of expired buprenorphine in Dr. Rosenfeld's study until in-date buprenorphine is available for purchase. A performance standard is not a departure from Guide, and its use is 